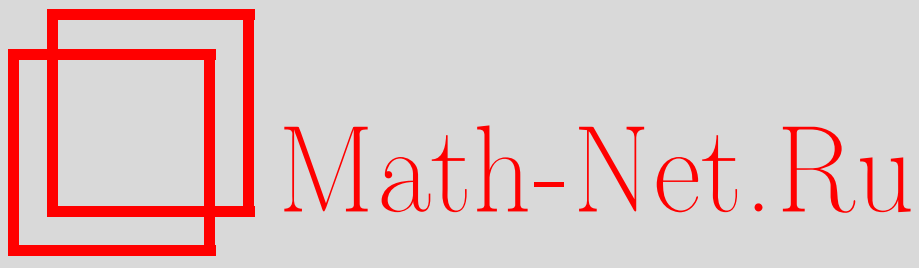

Г. Г. Лаптев, Отсутствие глобальных положительных решений систем полулинейных эллиптических неравенств в конусах, Изв. РАН. Сер. матем., 2000, том 64, выпуск 6, 107-124

DOI: https://doi.org/10.4213/im313

Использование Общероссийского математического портала Math-Net.Ru подразумевает, что вы прочитали и согласны с пользовательским соглашением http://www . mathnet.ru/rus/agreement

Параметры загрузки:

IP : 54.198 .187 .58

26 апреля 2023 г., 18:15:47 
УДК 517.9

\author{
Г.Г. Лаптев
}

\title{
Отсутствие глобальных положительных решений систем полулинейных эллиптических неравенств в конусах
}

\footnotetext{
Пусть $K$ - конус в пространстве $\mathbb{R}^{N}, N \geqslant 2$. В работе устанавливаются условия отсутствия глобальных неотрицательных нетривиальных решений полулинейных эллиптических неравенств и систем неравенств вида

$$
-\operatorname{div}\left(|x|^{\alpha} D u\right) \geqslant|x|^{\beta} u^{q},\left.\quad u\right|_{\partial K}=0 .
$$

Найден критический показатель $q^{*}$, разделяющий области существования и отсутствия указанных решений. Доказано отсутствие решения в предельном случае $q=q^{*}$. Для исследования задачи применяется метод умножения на специальный множитель с последующим интегрированием.

Библиография: 18 наименований.
}

\section{Введение}

В настояшее время большой интерес вызывают вопросы отсутствия глобальных неотрицательных (нетривиальных) решений квазилинейных эллиптических уравнений и неравенств. При этом основное внимание уделяется изучению уравнений и неравенств во всем пространстве $\mathbb{R}^{N}$ (см. [1]-[8]) и в конических областях (конуcax) (см. [9]-[14] и литературу там). Напомним, что конусом называется множество $K \in \mathbb{R}^{N}$, содержащее вместе с любой своей точкой $x \in K$ также все точки вида $\lambda x$, где $\lambda \in \mathbb{R}_{+}(\lambda>0)$. Под глобальным понимается решение, определенное для всех $x \in \mathbb{R}^{N}(x \in K)$, но не обязательно интегрируемое или ограниченное на всем $\mathbb{R}^{N}(K)$.

Классическим примером является задача

$$
-\Delta u \geqslant u^{q}, \quad u \geqslant 0, \quad \text { в } \mathbb{R}^{N}, \quad N \geqslant 2,
$$

где $\Delta$ - оператор Лапласа. Это неравенство не имеет глобального, отличного от нулевого решения [2], [5], [6], если $1<q \leqslant \frac{N}{N-2}$. Однако для $q>\frac{N}{N-2}$ требуемым решением служит функция

$$
u(x)=\frac{\varepsilon}{\left(1+|x|^{2}\right)^{1 /(q-1)}}
$$

при малых $\varepsilon>0$.

Аналогичный результат для конусов [9], [11] будет приведен в $\S 2$, где рассматривается соответствуюшая модельная задача.

В данной работе исследуется отсутствие локально ограниченных глобальных неотрищательных решений в конусах неравенств (§ 3$)$ вида

$$
-\operatorname{div}\left(|x|^{\alpha} D u\right) \geqslant|x|^{\beta} u^{q}, \quad \beta \geqslant \alpha \geqslant 0,
$$

Работа выполнена при финансовой поддержке INTAS (проект 96-1060) и Программы поддержки ведущих научных школ (проект 96-15-96102).

(C) Г.Г. ЛАПтев, 2000 
а также систем $(\S 4)$

$$
\left\{\begin{array}{l}
-\Delta u \geqslant v^{q} \\
-\Delta v \geqslant u^{p}
\end{array}\right.
$$

Результаты $\S 4$ могут быть обобщены на системы неравенств типа (0.2).

Предлагаемый подход не предполагает радиальности решений и не использует принципа сравнения и других утверждений, характерных для теории эллиптических уравнений и неравенств второго порядка. Важной особенностью является относительная простота доказательства отсутствия решения для предельного показателя роста нелинейного члена $u^{q}$.

Частично идеи доказательств заимствованы из теории емкости [15]-[17] и ее приложений к изучению отсутствия решений во всем пространстве $\mathbb{R}^{N}[2]-[6],[18]$.

\section{§ 1. Основные обозначения}

Пусть $K_{\omega}$ - область на единичной сфере $S^{N-1} \subset \mathbb{R}^{N}, N \geqslant 2$, с достаточно гладкой границей $\partial K_{\omega}$. Конусом $K$ называется множество

$$
K=\left\{x=x(r, \omega): 0<r<+\infty, \omega \in K_{\omega}\right\}
$$

где $(r, \omega)$ - сферические координаты точки $x$ в $\mathbb{R}^{N}$. Боковую поверхность конуса обозначаем $\partial K$.

В проводимых оценках через $c$ и $C$ обозначаются константы, точные значения которых для нас несущественны.

Под $L_{q, \text { loc }}(K), q \geqslant 1$, понимается пространство определенных на $K$ измеримых функций, суммируемых со степенью $q$ по любому (измеримому) ограниченному подмножеству $\Omega \subset K$; под $L_{\infty, \text { loc }}(K)$ - пространство локально существенно ограниченных функций. Для описания дифференциальных свойств применяются пространства Соболева

$$
\begin{aligned}
& W_{q, \mathrm{loc}}^{1}(K) \equiv\left\{u \in L_{q, \mathrm{loc}}(K):|D u| \in L_{q, \mathrm{loc}}(K)\right\} \\
& W_{q, \mathrm{loc}}^{2}(K) \equiv\left\{u \in L_{q, \mathrm{loc}}(K):\left|D^{2} u\right| \in L_{q, \mathrm{loc}}(K)\right\},
\end{aligned}
$$

где

$$
\begin{array}{rlrl}
D u & =\left(\frac{\partial u}{\partial x_{i}}\right), i=\overline{1, N} ; & & |D u|=\left(\sum_{i=1}^{N}\left(\frac{\partial u}{\partial x_{i}}\right)^{2}\right)^{1 / 2} ; \\
D^{2} u & =\left(\frac{\partial^{2} u}{\partial x_{i} \partial x_{j}}\right), \quad i, j=\overline{1, N} ; & \left|D^{2} u\right|=\left(\sum_{i=1}^{N} \sum_{j=1}^{N}\left(\frac{\partial^{2} u}{\partial x_{i} \partial x_{j}}\right)^{2}\right)^{1 / 2} .
\end{array}
$$

Если $u \in W_{q, \text { loc }}^{1}(K), \varphi \in W_{q^{\prime}, \text { loc }}^{1}(K)$ и $\frac{1}{q}+\frac{1}{q^{\prime}}=1$, то через $D u D \varphi$ обозначается сумма $\sum_{i=1}^{N} \frac{\partial u}{\partial x_{i}} \frac{\partial \varphi}{\partial x_{i}}$. Выражение $\operatorname{supp} \varphi$ обозначает носитель функции $\varphi$, т.е. множество $\{x \in K: \varphi(x) \neq 0\}$.

Напомним, что оператор Лапласа $\Delta$ в сферических координатах $(r, \omega)$ имеет вид

$$
\Delta=\frac{1}{r^{N-1}} \frac{\partial}{\partial r}\left(r^{N-1} \frac{\partial}{\partial r}\right)+\frac{1}{r^{2}} \Delta_{\omega}=\frac{\partial^{2}}{\partial r^{2}}+\frac{N-1}{r} \frac{\partial}{\partial r}+\frac{1}{r^{2}} \Delta_{\omega}
$$

где $\Delta_{\omega}$ - оператор Бельтрами-Лапласа на единичной сфере $S^{N-1} \subset \mathbb{R}^{N}$. 
В дальнейшем постоянно используются наименьшее собственное значение $\lambda_{\omega} \equiv$ $\lambda_{1}\left(K_{\omega}\right)>0$ и соответствуюшая собственная функция $\Phi(\omega)$ оператора $\Delta_{\omega}$, являющиеся решением задачи

$$
\left\{\begin{array}{l}
\Delta_{\omega} \Phi+\lambda \Phi=0 \quad \text { в } K_{\omega} \\
\left.\Phi\right|_{\partial K_{\omega}}=0 .
\end{array}\right.
$$

Хорошо известно, что $\Phi(\omega)>0$ для $\omega \in K_{\omega}$. Предполагается, что $\Phi(\omega) \leqslant 1$.

Собственное значение $\lambda_{\omega}$ наряду с размерностью $N$ является основным параметром, определяюшим существование или отсутствие решений в конусе $K$.

\section{§ 2. Модельная задача}

Рассмотрим проблему отсутствия нетривиальных неотрицательных слабых решений задачи

$$
\left\{\begin{array}{l}
-\Delta u \geqslant u^{q} \quad \text { в } K \\
\left.u\right|_{\partial K}=0 .
\end{array}\right.
$$

ОПРЕДЕЛЕНИЕ 2.1. ФУнкция $u(x) \in W_{2, \text { loc }}^{1}(K) \cap L_{\infty, \text { loc }}(K),\left.u\right|_{\partial K}=0$, называется слабы.м решением задачи (2.1), если для любой неотрицательной функции $\varphi(x) \equiv \varphi(r, \omega) \in W_{2}^{1}(K),\left.\varphi\right|_{\text {д }}=0$, финитной по переменной $r$, выполнено интегральное неравенство

$$
\int_{K} D u D \varphi d x \geqslant \int_{K} u^{q} \varphi d x .
$$

Цель данного параграфа состоит в нахождении степени $q^{*}>1$ такой, что при $1<q \leqslant q^{*}$ не существует неотрицательного нетривиального глобального слабого решения задачи (2.1).

Приведем пример решения задачи (2.1) при некоторых значениях $q$. Его отличием от известных аналогичных примеров для уравнений $[9],[11]$ является ограниченность функции $u(x)$ и ее производных в нуле.

ЛЕмма 2.1. Функиия

$$
u(x) \equiv u(r, \omega)=\frac{\varepsilon}{\left(1+r^{2}\right)^{1 /(q-1)}} \Phi(\omega)
$$

служит решением задачи (2.1) при достаточно малом $\varepsilon=\varepsilon(q)>0$ для любого

$$
q>q^{*}=1+\frac{2}{s^{*}} .
$$

Здес

$$
s^{*}=\frac{N-2}{2}+\sqrt{\left(\frac{N-2}{2}\right)^{2}+\lambda_{\omega}},
$$

$\Phi(\omega)$ - решение задачи $(1.1), \lambda_{\omega}$ - соответствующее наименьиее собственное значение.

ДокАЗАТЕЛЬСтво. Обозначим

$$
R(r)=\frac{\varepsilon}{\left(1+|r|^{2}\right)^{1 /(q-1)}} .
$$


Тогда $u=R(r) \Phi(\omega)$. Используя выражение для оператора Лапласа в сферических координатах и учитывая, что $\Delta_{\omega} \Phi=-\lambda_{\omega} \Phi$, получим

$$
-\Delta u=-\left(R^{\prime \prime} \Phi+\frac{N-1}{r} R^{\prime} \Phi+\frac{1}{r^{2}} R \Delta_{\omega} \Phi\right)=-\Phi\left(R^{\prime \prime}+\frac{N-1}{r} R^{\prime}-\frac{\lambda_{\omega}}{r^{2}} R\right) .
$$

Подставляем $R, R^{\prime}$ и $R^{\prime \prime}$, имеем

$$
-\Delta u=\frac{\varepsilon \Phi(\omega)}{\left(1+r^{2}\right)^{1 /(q-1)+1}}\left\{\frac{2 N}{q-1}-\frac{4}{q-1}\left(\frac{1}{q-1}+1\right) \frac{r^{2}}{1+r^{2}}+\lambda_{\omega} \frac{1+r^{2}}{r^{2}}\right\} .
$$

Легко проверить, что для всех $r>0$ выражение в фигурных скобках равномерно положительно:

$$
\frac{2 N}{q-1}-\frac{4 q}{(q-1)^{2}} \frac{r^{2}}{1+r^{2}}+\frac{\lambda_{\omega}}{r^{2}}+\lambda_{\omega}>\frac{2 N}{q-1}-\frac{4 q}{(q-1)^{2}}+\lambda_{\omega}>\delta(q)>0
$$

для фиксированного $q>1+\frac{2}{s^{*}}$.

Отсюда с учетом приведенного вьше выражения для $-\Delta u$ получаем

$$
-\Delta u>\frac{\varepsilon \Phi(\omega)}{\left(1+r^{2}\right)^{q /(q-1)}} \delta(q) \geqslant \frac{\varepsilon^{q} \Phi^{q}(\omega)}{\left(1+r^{2}\right)^{q /(q-1)}}=R^{q}(r) \Phi^{q}(\omega)=u^{q},
$$

т.е.

$$
-\Delta u \geqslant u^{q} .
$$

Здесь использовано условие $\Phi(\omega) \leqslant 1$, так что $\Phi(\omega) \geqslant \Phi^{q}(\omega)$, и выбрано число $\varepsilon=\delta^{1 /(q-1)}(q)>0$. Как видно, введенная функция $u(x) \in C^{2}(K) \cap L_{\infty}(K)$ и удовлетворяет неравенству (2.1) почти всюду в $K$. Достаточно очевидно, что всякое такое решение этого неравенства одновременно является слабым решением. Лемма доказана.

Основным утверждением настоящего параграфа является

TEOPEMA 2.1. Прu

$$
1<q \leqslant q^{*}=1+\frac{2}{s^{*}}
$$

задача (2.1) не имеет нетривиального неотрицательного глобального слабого решения. Здесь $s^{*}$ определено в (2.2).

ЗАмЕчАниЕ 2.1. Для случая уравнения аналоги этой теоремы имеются в работах [9], [11].

ДокАЗАТЕЛЬСТво. Для доказательства используется традиционный метод умножения неравенства (2.1) на специальный множитель с последуюшим интегрированием. Например, в работах [6], [2] для исследования задачи во всем пространстве $\mathbb{R}^{N}$ в качестве такого множителя берется функция $\frac{\varphi(x)}{u^{\alpha}(x)}$, где $u(x)$ - искомое решение, $\alpha>0, \varphi(x)$ - неотрицательная срезающая функция в $\mathbb{R}^{N}: \varphi(x) \equiv 1$ при $|x| \leqslant \rho, \varphi(x) \equiv 0$ при $|x| \geqslant 2 \rho, \varphi(x) \in C^{\infty}\left(\mathbb{R}^{N}\right), \rho$-некоторое число. В указанных работах задача сводится к вычислению $2 q^{\prime}$-емкости пространства $\mathbb{R}^{N}$.

В случае, когда областью является конус, этот подход сталкивается с сушественными трудностями, поэтому предлагается другой метод. В настоящей работе в качестве упомянутого множителя берется специальным образом выбранная функция $\varphi(x)$ без множителя $\frac{1}{u^{\alpha}(x)}$, после чего с помощью интегрирования по частям оператор $\Delta$ переносится с решения $u(x)$ на функцию $\varphi(x)$. 
Далее основное внимание уделяется оценке интегралов вида

$$
\int_{\operatorname{supp}|\Delta \varphi|} \frac{|\Delta \varphi|^{q^{\prime}}}{\varphi^{q^{\prime}-1}} d x
$$

где $\frac{1}{q}+\frac{1}{q^{\prime}}=1, \operatorname{supp}|\Delta \varphi|-$ носитель $|\Delta \varphi|$.

Для этого явно строится последовательность функций $\varphi_{\rho} \in W_{1}^{2}(K), \rho \rightarrow \infty$, удовлетворяющих некоторым дополнительным требованиям. После чего находятся условия на $q$, при которых указанные выше интегралы стремятся к нулю при $\rho \rightarrow \infty$. Эти условия и определяют отсутствие нетривиального глобального решения.

Итак, пусть $u \in W_{2, \text { loc }}^{1}(K) \cap L_{\infty, \text { loc }}(K)$ - слабое решение задачи $(2.1)$ с $1<$ $q \leqslant q^{*}$. Наша цель - показать, что тогда $u \equiv 0$. Согласно определению 2.1 слабого решения для любой неотрицательной функции $\varphi(x) \equiv \varphi(r, \omega) \in W_{2}^{1}(K)$, $\left.\varphi\right|_{\partial K}=0$, финитной по переменной $r$, справедливо соотношение

$$
\int_{K} u^{q} \varphi d x \leqslant \int_{K} D u D \varphi d x
$$

Предположим дополнительно, что функция $\varphi \in W_{1}^{2}(K) \cap W_{2}^{1}(K)$. Тогда, интегрируя правую часть (2.3) по частям, с учетом условия $\left.u\right|_{\partial K}=0$ получаем

$$
\int_{K} D u D \varphi d x=-\int_{K} u \Delta \varphi d x
$$

вследствие чего неравенство (2.3) принимает вид

$$
\int_{K} u^{q} \varphi d x \leqslant-\int_{K} u \Delta \varphi d x
$$

Для оценки правой части применим неравенство Юнга с параметром:

$$
\begin{aligned}
-\int_{K} u \Delta \varphi d x & =-\int_{\operatorname{supp}|\Delta \varphi|} u \Delta \varphi d x=-\int_{\operatorname{supp}|\Delta \varphi|} u \varphi^{1 / q} \frac{\Delta \varphi}{\varphi^{1 / q}} d x \\
& \leqslant \varepsilon \int_{\operatorname{supp}|\Delta \varphi|} u^{q} \varphi d x+\frac{1}{\varepsilon^{q^{\prime}-1}} \int_{\operatorname{supp}|\Delta \varphi|} \frac{|\Delta \varphi|^{q^{\prime}}}{\varphi^{q^{\prime}-1}} d x
\end{aligned}
$$

откуда при $\varepsilon=\frac{1}{2}$ получим

$$
\int_{\operatorname{supp} \varphi} u^{q} \varphi d x \leqslant c_{0} \int_{\operatorname{supp}|\Delta \varphi|} \frac{|\Delta \varphi|^{q^{\prime}}}{\varphi^{q^{\prime}-1}} d x
$$

где $c_{0}=2^{q^{\prime}}-$ не зависящая от $u$ и $\varphi$ постоянная.

Теперь необходимо построить последовательность функций $\varphi_{\rho}, \rho \rightarrow \infty$, удовлетворяющую следующим условиям:

1) $\varphi_{\rho} \stackrel{\text { п.в. }}{\longrightarrow} \varphi_{\infty}>0$ п.в. в $K ; \varphi_{\rho}=\varphi_{\infty}$ при $0<r<\rho$;

2) $\left.\varphi_{\rho}\right|_{\partial K}=0$

3) $\int_{\operatorname{supp}}\left|\Delta \varphi_{\rho}\right| \frac{\left|\Delta \varphi_{\rho}\right|^{q^{\prime}}}{\varphi_{\rho}^{q^{\prime}-1}} d x \rightarrow 0$ при $\rho \rightarrow \infty$.

Здесь $\varphi_{\infty} \in W_{1, \text { lос }}^{2}(K) \cap W_{2, \text { lос }}^{1}(K)$ - некоторая фиксированная функция. 
Тогда, подставляя в $(2.5) \varphi=\varphi_{\rho}$, будем иметь

$$
\int_{K \cap\{0<r<\rho\}} u^{q} \varphi_{\infty} d x \leqslant \int_{\operatorname{supp} \varphi_{\rho}} u^{q} \varphi_{\rho} d x \rightarrow 0 \text { при } \rho \rightarrow \infty,
$$

откуда в пределе получаем

$$
\int_{K} u^{q} \varphi_{\infty} d x=0
$$

что в силу неравенства $\varphi_{\infty}>0$ п.в. в $K$ означает $u \equiv 0$ в $K$, т.е. отсутствие глобального неотрицательного нетривиального решения.

Прервем доказательство теоремы 2.1 с целью построения последовательности $\varphi_{\rho}$ с указанными свойствами.

Пусть заданы числа $s>0, p>1$. Рассмотрим семейство функций

$$
\varphi_{\rho}(r, \omega)=R_{\rho}(r) \Phi(\omega),
$$

где $\Phi(\omega)$ - решение задачи (1.1), а функция $R_{\rho}(r)$ задается равенствами

$$
R_{\rho}(r)= \begin{cases}r^{s}, & 0 \leqslant r \leqslant \rho ; \\ \psi^{2 p}(r), & \rho<r<2 \rho \\ 0, & r \geqslant 2 \rho .\end{cases}
$$

Здесь

$$
\psi(r)=-\left(\frac{s}{2 p}+1\right) \rho^{\frac{s}{2 p}-2} r^{2}+\left(3 \frac{s}{2 p}+2\right) \rho^{\frac{s}{2 p}-1} r-2 \frac{s}{2 p} \rho^{\frac{s}{2 p}} .
$$

Отметим, что $\left.\varphi_{\rho}\right|_{\partial K}=0$ (для всех $0<r<2 \rho$ ) за счет множителя $\Phi(\omega)$.

Докажем некоторые свойства функции $\varphi_{\rho}$. Поскольку последуюшие действия носят чисто технический характер, будем опускать промежуточные выкладки.

ЛЕмма 2.2. Функиия $\varphi_{\rho}$ принадлежит $W_{1}^{2}(K) \cap W_{2}^{1}(K), \varphi_{\rho}=\varphi_{\infty}>0$ n.в. для $0<r<\rho$, әде $\varphi_{\infty}=r^{s} \Phi(\omega)$.

ДоКАЗАТЕЛЬСТво. Функция $R_{\rho}(r)$ является гладкой в интервалах $0<r<\rho$ и $\rho<r<2 \rho$. Функция $\psi(r)$ введена для того, чтобы на участке $\rho<r<2 \rho$ функция $r^{s} \Phi(\omega)$ срезалась гладким образом $\left(\varphi_{\rho} \in W_{1}^{2}(K)\right)$, достигая финитности по переменной $r$. Конкретный многочлен $\psi(r)$ приведен с целью наиболее наглядного и явного выгисления получающихся интегралов.

Убедимся, что функции $R_{\rho}(r)$ и $R_{\rho}^{\prime}(r)$ непрерывны в точках стыка $r=\rho$ и $r=2 \rho$. Это приводит к системе равенств:

$$
\left\{\begin{array} { l } 
{ \psi ^ { 2 p } ( \rho ) = \rho ^ { s } } \\
{ 2 p \psi ^ { 2 p - 1 } ( \rho ) \psi ^ { \prime } ( \rho ) = s \rho ^ { s - 1 } } \\
{ \psi ^ { 2 p } ( 2 \rho ) = 0 } \\
{ 2 p \psi ^ { 2 p - 1 } ( 2 \rho ) \psi ^ { \prime } ( 2 \rho ) = 0 , }
\end{array} \Leftrightarrow \left\{\begin{array}{l}
\psi(\rho)=\rho^{\frac{s}{2 p}} \\
\psi^{\prime}(\rho)=\frac{s}{2 p} \rho^{\frac{s}{2 p}}-1 \\
\psi(2 \rho)=0
\end{array}\right.\right.
$$

Справедливость данных равенств для введенной функции $\psi(r)$ проверяется прямой подстановкой. Собственно говоря, функция $\psi(r)$ подбиралась так, чтобы выполнялись указанные соотношения.

Отметим, что $R_{\rho}^{\prime}=s r^{s-1}$ и $R_{\rho}^{\prime \prime}=s(s-1) r^{s-2}$ для $0<r<\rho$, и так как $s>0$, то отсюда следует

$$
\varphi_{\rho} \in W_{1}^{2}(K \cap\{0<r<1\}) \cap W_{2}^{1}(K \cap\{0<r<1\}) .
$$


ЛЕмма 2.3. Для всех $\rho>1$ выполняется неравенство

$$
\int_{K \cap\{\rho<r<2 \rho\}} \frac{\left|\Delta \varphi_{\rho}\right|^{p}}{\varphi_{\rho}^{p-1}} d x \leqslant \frac{C}{\rho^{2 p-s-N}}
$$

где постоянная $C$ не зависит от $\rho$.

ДокаЗАТЕЛЬство. Записывая оператор Лапласа в сферической системе координат, для функции $\varphi_{\rho}=\psi^{2 p}(r) \Phi(\omega)$ в интервале $\rho<r<2 \rho$ получаем оценку

$$
\begin{aligned}
\left|\Delta \varphi_{\rho}\right|= & \left|\frac{\partial^{2} \varphi_{\rho}}{\partial r^{2}}+\frac{N-1}{r} \frac{\partial \varphi_{\rho}}{\partial r}+\frac{1}{r^{2}} \Delta_{\omega} \varphi_{\rho}\right| \\
= & \left|\left(\psi^{2 p}\right)^{\prime \prime} \Phi+\frac{N-1}{r}\left(\psi^{2 p}\right)^{\prime} \Phi-\lambda_{\omega} \frac{\psi^{2 p}}{r^{2}} \Phi\right| \\
= & \Phi(\omega) \mid 2 p \psi^{2 p-1}(r) \psi^{\prime \prime}(r)+2 p(2 p-1) \psi^{2 p-2}(r)\left(\psi^{\prime}(r)\right)^{2} \\
& \quad+\frac{N-1}{r} 2 p \psi^{2 p-1}(r) \psi^{\prime}(r)-\frac{\lambda_{\omega}}{r^{2}} \psi^{2 p}(r) \mid \\
\leqslant & c_{1} \Phi(\omega) \psi^{2 p-2}(r)\left\{\psi(r)\left|\psi^{\prime \prime}(r)\right|+\left|\psi^{\prime}(r)\right|^{2}+\frac{\psi(r)\left|\psi^{\prime}(r)\right|}{r}+\frac{\psi^{2}(r)}{r^{2}}\right\}
\end{aligned}
$$

где $c_{1}$ зависит от $p, N$ и $\lambda_{\omega}$, но не зависит от $r$ и $\rho$.

Из явного вида функции $\psi(r)$ для $\rho<r<2 \rho$ получаем

$$
|\psi(r)| \leqslant c_{2} \rho^{\frac{s}{2 p}}, \quad\left|\psi^{\prime}(r)\right| \leqslant c_{2} \rho^{\frac{s}{2 p}-1}, \quad\left|\psi^{\prime \prime}(r)\right| \leqslant c_{2} \rho^{\frac{s}{2 p}-2},
$$

где $c_{2}$ не зависит от $r$ и $\rho$.

Учитьвая, что $r>\rho$, можем закончить оценку $\left|\Delta \varphi_{\rho}\right|$ :

$$
\left|\Delta \varphi_{\rho}\right| \leqslant c_{3} \Phi(\omega) \psi^{2 p-2}(r) \rho^{\frac{s}{p}-2}=c_{3} \rho^{\frac{s}{p}-2} R_{\rho}^{1-\frac{1}{p}}(r) \Phi(\omega),
$$

где $c_{3}$ не зависит от $r$ и $\rho$.

Итак, для интеграла из формулировки леммы имеем

$$
\begin{aligned}
\int_{K \cap\{\rho<r<2 \rho\}} \frac{\left|\Delta \varphi_{\rho}\right|^{p}}{\varphi_{\rho}^{p-1}} d x & \leqslant \int_{K \cap\{\rho<r<2 \rho\}} \frac{c_{3}^{p} \rho^{s-2 p} R_{\rho}^{p-1}(r) \Phi^{p}(\omega)}{R_{\rho}^{p-1}(r) \Phi^{p-1}(\omega)} d x \\
& =c_{3}^{p} \rho^{s-2 p} \int_{K_{\omega}} \Phi(\omega) d \omega \int_{\rho}^{2 \rho} r^{N-1} d r \leqslant \frac{C}{\rho^{2 p-s-N}} .
\end{aligned}
$$

Лемма доказана.

Основная идея построения семейства $\varphi_{\rho}$ заключается в том, что при некотором значении $s=s_{*}$ функция $\varphi_{\rho}$ на множестве $K \cap\{0<r<\rho\}$ предполагается гармонической, т.е. удовлетворяющей уравнению $\Delta \varphi_{\rho}=0$. Это приводит к множителю $R_{\rho}(r)=r^{s}$. В следуюшем утверждении определяется конкретное значение $s_{*}$.

Лемма 2.4. Функиия $\varphi_{\rho}$ при

$$
s=s_{*}=-\frac{N-2}{2}+\sqrt{\left(\frac{N-2}{2}\right)^{2}+\lambda_{\omega}}
$$

является гармонической на множестве $K \cap\{0<r<\rho\}$. В частности, $\operatorname{supp}\left|\Delta \varphi_{\rho}\right|=K \cap\{\rho<r<2 \rho\}$. 
ДокАЗАТЕЛЬСТво. Записывая оператор Лапласа в сферической системе координат и учитьвая, что для $0<r<\rho$ по определению $\varphi_{\rho}=r^{s} \Phi(\omega)$, получим

$$
\begin{aligned}
\Delta \varphi_{\rho} & =\frac{\partial^{2} \varphi_{\rho}}{\partial r^{2}}+\frac{N-1}{r} \frac{\partial \varphi_{\rho}}{\partial r}+\frac{1}{r^{2}} \Delta_{\omega} \varphi_{\rho} \\
& =\left(r^{s}\right)^{\prime \prime} \Phi+\frac{N-1}{r}\left(r^{s}\right)^{\prime} \Phi+\frac{1}{r^{2}} r^{s} \Delta_{\omega} \Phi \\
& =s(s-1) r^{s-2} \Phi+\frac{N-1}{r} s r^{s-1} \Phi-\lambda_{\omega} r^{s-2} \Phi \\
& =r^{s-2} \Phi(\omega)\left\{s(s-1)+s(N-1)-\lambda_{\omega}\right\} \equiv 0,
\end{aligned}
$$

поскольку выражение в скобках обрашается в нуль при $s=s_{*}$, как показывает непосредственное вычисление (использующее определение числа $s_{*}$ ).

Продолжим доказательство теоремы 1. Обратимся к неравенству (2.5), справедливому, в частности, и для построенных выше функций $\varphi_{\rho}, \rho \rightarrow \infty(2.6)$. Положим в определении функции $\varphi_{\rho}$ степень $s=s_{*}\left(s_{*}\right.$ задано в лемме 2.4$)$. Для $\varphi=\varphi_{\rho}$ из (2.5) получим в соответствии с леммой 2.3

$$
\begin{aligned}
\int_{K \cap\{0<r<\rho\}} u^{q} \varphi_{\infty} d x & \leqslant \int_{\operatorname{supp} \varphi_{\rho}} u^{q} \varphi_{\rho} d x \\
& \leqslant c_{0} \int_{\operatorname{supp}\left|\Delta \varphi_{\rho}\right|} \frac{\left|\Delta \varphi_{\rho}\right|^{q^{\prime}}}{\varphi_{\rho}^{q^{\prime}-1}} d x \leqslant \frac{c_{0} C}{\rho^{2 q^{\prime}-s_{*}-N}} .
\end{aligned}
$$

Введем показатель

$$
q^{*}=1+\frac{2}{s^{*}}
$$

где $s^{*}=s_{*}+N-2$ определяется формулой $(2.2)$. Легко видеть, что для $1<q<q^{*}$ показатель $2 q^{\prime}-s_{*}-N>0$, поэтому вьполняется предельное соотношение

$$
\int_{K \cap\{0<r<\rho\}} u^{q} \varphi_{\infty} d x \rightarrow 0 \text { при } \rho \rightarrow \infty .
$$

Для $q=q^{*}$ имеем $2 q^{\prime}-s_{*}-N=0$, поэтому справедливо неравенство

$$
\int_{K \cap\{0<r<\rho\}} u^{q} \varphi_{\infty} d x \leqslant C \text { для всех } \rho \rightarrow \infty .
$$

Напомним, что постоянная $C$ здесь не зависит от $\rho$, так как взята из леммы 2.3 .

Поскольку в левых частях последних двух соотношений от $\rho$ зависит только область интегрирования, можем перейти к пределу при $\rho \rightarrow \infty$, что дает

$$
\begin{aligned}
& \int_{K} u^{q} \varphi_{\infty} d x=0 \text { при } 1<q<q^{*}, \\
& \int_{K} u^{q} \varphi_{\infty} d x \leqslant C \text { при } q=q^{*} .
\end{aligned}
$$

Первое равенство (поскольку $\varphi_{\infty}=r^{s_{*}} \Phi(\omega)>0$ п.в. в К) означает, что $u \equiv 0$. Это доказывает отсутствие глобального неотрицательного нетривиального решения для $q<q^{*}$. 
В предельном случае $q=q^{*}$ неравенство (2.7) гарантирует лишь глобальную суммируемость функции $u^{q} \varphi_{\infty}$ в $K$. Для доказательства отсутствия нетривиальных решений вернемся к оценке (2.4), но применим в ней вместо неравенства Юнга неравенство Гёльдера. Получим (для $\left.\varphi=\varphi_{\rho}\right)$

$$
\begin{aligned}
\int_{K} u^{q} \varphi_{\rho} d x & \leqslant-\int_{K} u \Delta \varphi_{\rho} d x \\
& \leqslant\left(\int_{\operatorname{supp}\left|\Delta \varphi_{\rho}\right|} u^{q} \varphi_{\rho} d x\right)^{1 / q}\left(\int_{\operatorname{supp}\left|\Delta \varphi_{\rho}\right|} \frac{\left|\Delta \varphi_{\rho}\right|^{q^{\prime}}}{\varphi_{\rho}^{q^{\prime}-1}} d x\right)^{1 / q^{\prime}},
\end{aligned}
$$

где $q=q^{*}$.

Используя явньй вид функции $\varphi_{\rho}$, получаем оценку $\varphi_{\rho}(r, \omega) \leqslant c_{4} \varphi_{\infty}(r, \omega)$ для всех $0<r<+\infty$ и всех $\rho \rightarrow \infty$ с постоянной $c_{4}$, не зависящей от $\rho$, откуда

$$
\int_{\operatorname{supp}\left|\Delta \varphi_{\rho}\right|} u^{q} \varphi_{\rho} d x \leqslant c_{4} \int_{\operatorname{supp}\left|\Delta \varphi_{\rho}\right|} u^{q} \varphi_{\infty} d x \leqslant c_{4} \int_{K \cap\{\rho<r<2 \rho\}} u^{q} \varphi_{\infty} d x .
$$

В то же время согласно (2.7) интеграл от $u^{q} \varphi_{\infty}$ по всему конусу $K$ ограничен, поэтому из обшей теории интеграла по бесконечной мере следует соотношение

$$
\int_{K \cap\{\rho<r<2 \rho\}} u^{q} \varphi_{\infty} d x=\varepsilon(\rho) \rightarrow 0 \text { при } \rho \rightarrow \infty .
$$

Тогда из неравенства (2.8) получим

$$
\int_{K \cap\{0<r<\rho\}} u^{q} \varphi_{\infty} d x \leqslant \varepsilon^{1 / q}(\rho) C^{1 / q^{\prime}} \rightarrow 0 \text { при } \rho \rightarrow \infty,
$$

т.е. в пределе

$$
\int_{K} u^{q} \varphi_{\infty} d x=0
$$

Так как $\varphi_{\infty}>0$ п.в. в $K$, то отсюда следует, что $u \equiv 0$. Это доказывает отсутствие глобального нетривиального решения и в случае $q=q^{*}$.

ЗАмЕчАниЕ 2.2. В данной теореме, как и во всей работе, рассматриваются только локально ограниченные в конусе $K$ решения неравенств. В частности, не допускается неограниченная особенность решения в нуле. Покажем, как ограниченность влияет на сушествование и отсутствие решений для случая всего пространства $\mathbb{R}^{N}$. Напомним некоторые утверждения.

ПРЕДЛОЖЕНИЕ 2.1 [2], [6]. Неравенство

$$
-\Delta u \geqslant u^{q}, \quad u>0, \quad x \in \mathbb{R}^{N},
$$

не имеет глобального классического решения при $q \leqslant \frac{N}{N-2}$.

ПРЕДЛОЖЕНИЕ 2.2 [7]. Уравнение

$$
-\Delta u=u^{q}, \quad u>0, \quad x \in \mathbb{R}^{N},
$$

не имеет глобального классического решения при $q<\frac{N+2}{N-2}$.

Как видно, критические показатели для уравнений и неравенств в случае классических решений не совпадают. В то же время, если допустить сингулярность решения в одной точке, можно высказать следующее утверждение. 
ПРЕДЛОЖЕНИЕ 2.3. ФунКция

$$
u(x)=\frac{\varepsilon}{|x|^{s}}, \quad \text { əде } \quad s=\frac{2}{q-1}, \quad \varepsilon=(s(N-s-2))^{1 /(q-1)},
$$

является решением из $C^{2}\left(\mathbb{R}^{N} \backslash\{0\}\right)$ уравнения $-\Delta u=u^{q}, \quad u>0, \quad x \in \mathbb{R}^{N}$, прu $q>\frac{N}{N-2}$.

Таким образом, критический показатель отсутствия решений, допускаюших сингулярность в нуле, не превосходит критического показателя отсутствия классических решений соответствуюшего неравенства.

Аналогичньй факт справедлив и для задач в конусах.

ПрЕДЛОЖЕНИЕ 2.4 [9]. Уравнение

$$
-\Delta u=u^{q}, \quad u \geqslant 0, \quad x \in K \subset \mathbb{R}^{N},
$$

не имеет глобального нетривиального решения из $C^{2}(K) \cap C(\bar{K} \backslash\{0\})$, если

$$
1<q<1+\frac{2}{s^{*}}
$$

где s* определяется по формуле (2.2).

ПреДЛОЖЕНИЕ 2.5 [9]. Функиия

$$
u(r, \omega)=\frac{1}{r^{2 /(q-1)}} \alpha(\omega)
$$

әде $\alpha(\omega)$ - решение уравнения

$$
\Delta_{\omega} \alpha(\omega)-\frac{2}{q-1}\left(N-2-\frac{2}{q-1}\right) \alpha(\omega)+\alpha^{q}(\omega)=0, \quad \omega \in K_{\omega},\left.\quad \alpha(\omega)\right|_{\partial K_{\omega}}=0,
$$

является глобальным решением из $C^{2}(K) \cap C(\bar{K} \backslash\{0\})$ уравнения

$$
-\Delta u=u^{q}, \quad u \geqslant 0, \quad x \in K,
$$

npu

$$
1+\frac{2}{s^{*}}<q< \begin{cases}+\infty, & N=2,3, \\ \frac{N+1}{N-3}, & N>3 .\end{cases}
$$

Сравнение этого предложения с теоремой 2.1 и леммой 2.1 показывает, что критические показатели для уравнений и неравенств совпадают, если допустить сингулярность решения уравнения в нуле.

ЗАмЕчАнИЕ 2.3. Предлагаемый подход применим и к доказательству отсутствия локально ограниченных решений во всем пространстве $\mathbb{R}^{N}$. При этом нужно брать $\Phi(\omega) \equiv 1$ и соответствуюшее $\lambda_{\omega}=0$. Тогда $s_{*}=0$ и получаюшаяся функция $\varphi_{\rho}(x)$ сходна со срезающей функцией из работ [5], [2].

Необходимо уточнить, что непосредственное использование теоремы 2.1 в "предельном" случае $K_{\omega} \rightarrow S^{N-1}$ не приводит к условиям, установленным для всего $\mathbb{R}^{N}$. Рассмотрим подробнее случай $N=2$. Тогда $K_{\omega}=(0, l)$ - интервал (дуга окружности). В предельном случае окружности $l=2 \pi$ задача (1.1) принимает вид

$$
\frac{d^{2} \Phi}{d \omega^{2}}+\lambda \Phi=0, \quad \Phi(0)=\Phi(2 \pi)=0 .
$$


Ее неотрицательным решением является функция $\Phi(\omega)=\sin \frac{\omega}{2}$, что соответствует наименьшему собственному значению $\lambda_{\omega}=\frac{1}{4}$.

Тогда по теореме 2.1 критический показатель равен

$$
q^{*}=1+\frac{2}{s^{*}}=1+\frac{2}{\sqrt{\lambda_{\omega}}}=5,
$$

в то время как аналогичный показатель, найденный из предложения 2.1 , есть $\infty$.

\section{§ 3. Неравенство общего вида}

Применим описанный в предыдушем параграфе метод к исследованию отсутствия неотрицательных нетривиальных слабых решений задачи

$$
\left\{\begin{array}{l}
-\operatorname{div}\left(|x|^{\alpha} D u\right) \geqslant|x|^{\beta} u^{q}, \quad 0 \leqslant \alpha \leqslant \beta \\
\left.u\right|_{\partial K}=0
\end{array}\right.
$$

ОПРЕДЕЛЕниЕ 3.1 . Функция $u(x) \in W_{2, \mathrm{loc}}^{1}(K) \cap L_{\infty, \mathrm{loc}}(K),\left.u\right|_{\partial K}=0$, называется слабым решением задачи (3.1), если для любой неотрицательной функции $\varphi(x) \equiv \varphi(r, \omega) \in W_{2}^{1}(K),\left.\varphi\right|_{\partial K}=0$, финитной по переменной $r$, выполнено интегральное неравенство

$$
\int_{K}|x|^{\alpha} D u D \varphi d x \geqslant \int_{K}|x|^{\beta} u^{q} \varphi d x
$$

Для упрощения последующих выкладок введем оператор

$$
A_{\alpha} \varphi=\operatorname{div}\left(|x|^{\alpha} D \varphi\right) .
$$

При $\alpha=0$ имеем $A_{0} \varphi=\Delta \varphi$.

Рассмотрим семейство функций $\varphi_{\rho}$, определенных формулами (2.6). Установим аналоги лемм 2.3 и 2.4 , соответствуюшие задаче (3.1).

Лемма 3.1. Для всех $\rho>1$ выполняется неравенство

$$
\int_{K \cap\{\rho<r<2 \rho\}} \frac{\left|A_{\alpha} \varphi_{\rho}\right|^{p}}{|x|^{\beta(p-1)} \varphi_{\rho}^{p-1}} d x \leqslant \frac{C}{\rho^{(2+\beta-\alpha) p-s-\beta-N}},
$$

где постоянная $C$ не зависит от $\rho$; оператор $A_{\alpha}$ определен формулой (3.2). Здесь $p>1, s>0$ - заданные числа.

ДокАЗАТЕЛЬСтво. Запишем оператор $A_{\alpha} \varphi$ в сферической системе координат:

$$
A_{\alpha} \varphi_{\rho} \equiv \operatorname{div}\left(|x|^{\alpha} D \varphi_{\rho}\right)=r^{\alpha}\left(\frac{\partial^{2} \varphi_{\rho}}{\partial r^{2}}+\frac{\alpha+N-1}{r} \frac{\partial \varphi_{\rho}}{\partial r}+\frac{1}{r^{2}} \Delta_{\omega} \varphi_{\rho}\right) .
$$

Далее, как и в лемме 2.3 , для $\rho<r<2 \rho$ с учетом явного вида функции $\varphi_{\rho}$ получаем оценку

$$
\left|A_{\alpha} \varphi_{\rho}\right| \leqslant c_{3} r^{\alpha} \rho^{\frac{s}{p}-2} R_{\rho}^{1-\frac{1}{p}}(r) \Phi(\omega) \text { для } \rho<r<2 \rho,
$$

где $c_{3}$ не зависит от $r$ и $\rho$. Отсюда

$$
\begin{array}{r}
\int_{K \cap\{\rho<r<2 \rho\}} \frac{\left|A_{\alpha} \varphi_{\rho}\right|^{p}}{|x|^{\beta(p-1)} \varphi_{\rho}^{p-1}} d x \leqslant \int_{K \cap\{\rho<r<2 \rho\}} \frac{c_{3}^{p} r^{\alpha p} \rho^{s-2 p} R_{\rho}^{p-1}(r) \Phi^{p}(\omega)}{r^{\beta(p-1)} R_{\rho}^{p-1}(r) \Phi^{p-1}(\omega)} d x \\
=c_{3}^{p} \rho^{s-2 p} \int_{K_{\omega}} \Phi(\omega) d \omega \int_{\rho}^{2 \rho} r^{\alpha p-\beta(p-1)+N-1} d r \leqslant \frac{C}{\rho^{(2+\beta-\alpha) p-s-\beta-N}} .
\end{array}
$$

Лемма доказана.

Доказательство следующего утверждения проводится в полной аналогии с доказательством леммы 2.4 . 
ЛЕмма 3.2. Функиия $\varphi_{\rho} \partial л я$

$$
s=s_{* \alpha}=-\frac{\alpha+N-2}{2}+\sqrt{\left(\frac{\alpha+N-2}{2}\right)^{2}+\lambda_{\omega}}
$$

яв.яется решением уравнения $A_{\alpha} \varphi_{\rho}=0$ на множестве $K \cap\{0<r<\rho\} . B$ частности, supp $\left|A_{\alpha} \varphi_{\rho}\right|=K \cap\{\rho<r<2 \rho\}$. Здесь оператор $A_{\alpha} \varphi$ определен формулой (3.2).

Условия отсутствия глобальных неотрищательных решений задачи (3.1) дает

TeOpema 3.1. Прu

$$
1<q \leqslant q_{\alpha \beta}^{*}=1+\frac{2+\beta-\alpha}{s_{\alpha}^{*}},
$$

2дe

$$
s_{\alpha}^{*}=\frac{\alpha+N-2}{2}+\sqrt{\left(\frac{\alpha+N-2}{2}\right)^{2}+\lambda_{\omega}}=s_{* \alpha}+\alpha+N-2,
$$

задача (3.1) не имеет глобального неотричательного нетривиального слабого решения.

ДоКАЗАТЕльСТво следует схеме доказательства теоремы 2.1 .

Пусть $u(x)$ - слабое решение задачи (3.1). Тогда для любой финитной по переменной $r$ функции $\varphi(r, \omega) \in W_{1}^{2}(K) \cap W_{2}^{1}(K),\left.\varphi\right|_{\partial K}=0$, с помощью интегрирования по частям из определения обобщенного решения получим

$$
\int_{K}|x|^{\beta} u^{q} \varphi d x \leqslant-\int_{K} u A_{\alpha} \varphi d x
$$

где $A_{\alpha} \varphi$ определен формулой (3.2). Применяя для оценки правой части неравенство Юнга с параметром, после приведения подобных членов будем иметь

$$
\int_{\operatorname{supp} \varphi}|x|^{\beta} u^{q} \varphi d x \leqslant c_{0} \int_{\operatorname{supp}\left|A_{\alpha} \varphi\right|} \frac{\left|A_{\alpha} \varphi\right|^{q^{\prime}}}{|x|^{\beta\left(q^{\prime}-1\right)} \varphi^{q^{\prime}-1}} d x
$$

где $c_{0}$ не зависит от $u$ и $\varphi$.

Для $\varphi=\varphi_{\rho}$ отсюда в соответствии с леммой 3.1 получаем

$$
\int_{K \cap\{0<r<\rho\}}|x|^{\beta} u^{q} \varphi_{\infty} d x \leqslant \int_{\operatorname{supp} \varphi_{\rho}}|x|^{\beta} u^{q} \varphi_{\rho} d x \leqslant \frac{c_{0} C}{\rho^{(2+\beta-\alpha) q^{\prime}-s_{* \alpha}-\beta-N}} .
$$

Введем параметр

$$
q_{\alpha \beta}^{*}=1+\frac{2+\beta-\alpha}{s_{\alpha}^{*}},
$$

где $s_{\alpha}^{*}=s_{* \alpha}+\alpha+N-2$ определено в формулировке теоремы, и перейдем в этом неравенстве при $1<q \leqslant q_{\alpha \beta}^{*}$ к пределу по $\rho \rightarrow \infty$, что даст

$$
\begin{aligned}
& \int_{K}|x|^{\beta} u^{q} \varphi_{\infty} d x=0 \text { при } q<q_{\alpha \beta}^{*}, \\
& \int_{K}|x|^{\beta} u^{q} \varphi_{\infty} d x \leqslant C \text { при } q=q_{\alpha \beta}^{*} .
\end{aligned}
$$


Эти соотношения означают, как в доказательстве теоремы 2.1 , отсутствие неотрицательных нетривиальных решений. Теорема доказана.

Результат теоремы 3.1 точен. Функция

$$
u(r, \omega)=\frac{\varepsilon}{\left(1+r^{2}\right)^{\gamma}} \Phi(\omega), \quad \gamma=\frac{\beta-\alpha+2}{2(q-1)},
$$

служит решением задачи (3.1) при малом $\varepsilon=\varepsilon(q)>0$ для любого

$$
q>q_{\alpha \beta}^{*}=1+\frac{2+\beta-\alpha}{s_{\alpha}^{*}},
$$

где $s_{\alpha}^{*}$ определяется формулой (3.3).

Чтобы убедиться в этом, запишем оператор $-A_{\alpha} u=-\operatorname{div}\left(|x|^{\alpha} D u\right)$ в сферической системе координат. Аналогично доказательству леммы 2.1 для определенной формулой $(3.4)$ функции $u(r, \omega)$ будем иметь

$$
-\operatorname{div}\left(|x|^{\alpha} D u\right)=\varepsilon \frac{r^{\alpha}}{\left(1+r^{2}\right)^{\gamma+1}} \Phi(\omega)\left\{2 \gamma(\alpha+N)-4 \gamma(\gamma+1) \frac{r^{2}}{1+r^{2}}+\frac{\lambda_{\omega}}{r^{2}}+\lambda_{\omega}\right\} .
$$

Легко проверить, что выражение в фигурных скобках равномерно положительно:

$2 \gamma(\alpha+N)-4 \gamma(\gamma+1) \frac{r^{2}}{1+r^{2}}+\frac{\lambda_{\omega}}{r^{2}}+\lambda_{\omega}>2 \gamma(\alpha+N)-4 \gamma(\gamma+1)+\lambda_{\omega}>\delta(q)>0$, для фиксированного $q>q_{\alpha \beta}^{*}$ (при $\gamma$, определенном в (3.4)).

Отсюда при $q>q_{\alpha \beta}^{*}$ с учетом неравенства $r^{\beta-\alpha} \leqslant\left(1+r^{2}\right)^{(\beta-\alpha) / 2}$ получим

$$
\begin{aligned}
-\operatorname{div}\left(|x|^{\alpha} D u\right) & \geqslant \varepsilon \Phi(\omega) \frac{r^{\alpha}}{\left(1+r^{2}\right)^{\gamma+1}} \delta(q) \\
& =\varepsilon \Phi(\omega) \frac{r^{\beta}}{\left(1+r^{2}\right)^{\gamma+1} r^{\beta-\alpha}} \delta(q) \geqslant \varepsilon \Phi(\omega) \frac{r^{\beta}}{\left(1+r^{2}\right)^{\gamma+1+(\beta-\alpha) / 2}} \delta(q) .
\end{aligned}
$$

Подставляя конкретное значение $\gamma$ из $(3.4)$ и используя оценку $\Phi(\omega) \geqslant \Phi^{q}(\omega)$, окончательно приходим к неравенству

$$
\begin{aligned}
-\operatorname{div}\left(|x|^{\alpha} D u\right) & \geqslant \varepsilon \Phi(\omega) \frac{r^{\beta}}{\left(1+r^{2}\right)^{\gamma q}} \delta(q) \geqslant r^{\beta} \frac{\varepsilon \delta(q)}{\left(1+r^{2}\right)^{\gamma q}} \Phi^{q}(\omega) \\
& =r^{\beta}\left(\frac{\varepsilon}{\left(1+r^{2}\right)^{\gamma}}\right)^{q} \Phi^{q}(\omega)=|x|^{\beta} u^{q},
\end{aligned}
$$

если положить $\varepsilon=\delta(q)^{1 /(q-1)}$. Тем самым показано, что функция $u(x)$ является решением задачи (3.1) при $q>q_{\alpha \beta}^{*}$.

Сравним критический показатель теоремы 3.1 с одним из результатов статьи [11] для уравнения

$$
-\Delta u-\sum_{i=1}^{N} a_{i}(x) \frac{\partial u}{\partial x_{i}}=|x|^{\sigma} u^{q},\left.\quad u\right|_{\partial K \backslash\{0\}}=0 .
$$

В наших обозначениях теорема 3.1 из [11] утверждает, что если

$$
\left|a_{i}(x)\right| \leqslant \frac{c}{|x|^{1+\varepsilon}}, \quad \varepsilon>0
$$


то эта задача не имеет неотрищательного нетривиального решения для

$$
1<q<q_{M}=1+\frac{2+\sigma}{s^{*}},
$$

где $s^{*}$ определено формулой (2.2).

Tak как

$$
\operatorname{div}\left(|x|^{\alpha} D u\right)=|x|^{\alpha}\left(\Delta u+\alpha \sum_{i=1}^{N} \frac{x_{i}}{|x|^{2}} \frac{\partial u}{\partial x_{i}}\right),
$$

то неравенство (3.1) для случая дважды дифференцируемых решений можно переписать в виде

$$
-\Delta u-\sum_{i=1}^{N} a_{i}(x) \frac{\partial u}{\partial x_{i}} \geqslant|x|^{\beta-\alpha} u^{q}
$$

где

$$
a_{i}(x)=\alpha \frac{x_{i}}{|x|^{2}}, \quad\left|a_{i}(x)\right| \leqslant \frac{c}{|x|} .
$$

По теореме 3.1 критический показатель равен

$$
q_{\alpha \beta}^{*}=1+\frac{2+\beta-\alpha}{s_{\alpha}^{*}}<q_{M}=1+\frac{2+\beta-\alpha}{s^{*}},
$$

где $s_{\alpha}^{*}$ определено в $(3.3)$ и $s_{\alpha}^{*}>s^{*}$.

Из приведенного сравнения очевидно, что незначительное изменение поведения функций $a_{i}(x)$ при $|x| \rightarrow \infty$ приводит к резкому отличию критических показателей. В частности, на них начинают влиять постоянные, входящие множителями в коэффициенты при младших производных.

\section{§ 4. Система неравенств}

Рассмотрим систему

$$
\begin{gathered}
\left\{\begin{array}{l}
-\Delta u \geqslant v^{q} \\
-\Delta v \geqslant u^{p} \quad \text { в } K,
\end{array}\right. \\
\left.u\right|_{\partial K}=0,\left.\quad v\right|_{\partial K}=0 .
\end{gathered}
$$

ОПРЕДЕЛЕНИЕ 4.1. Пара функций $u, v \in W_{2, \text { loc }}^{1}(K) \cap L_{\infty, \text { loc }}(K),\left.u\right|_{\partial K}=0$, $\left.v\right|_{\partial K}=0$, называется слабым решением задачи (4.1), если для любой неотрицательной функции $\varphi(x) \equiv \varphi(r, \omega) \in W_{2}^{1}(K),\left.\varphi\right|_{\partial K}=0$, финитной по переменной $r$, выполнены интегральные неравенства

$$
\begin{aligned}
& \int_{K} D u D \varphi d x \geqslant \int_{K} v^{q} \varphi d x \\
& \int_{K} D v D \varphi d x \geqslant \int_{K} u^{p} \varphi d x .
\end{aligned}
$$


ТеОрема 4.1. Задача (4.1) не имеет глобального неотрицательного нетривиального слабого решения, если

$$
\max \left\{\gamma_{1}, \gamma_{2}\right\} \geqslant \frac{s^{*}}{2},
$$

əде

$$
\gamma_{1}=\frac{q+1}{p q-1}, \quad \gamma_{2}=\frac{p+1}{p q-1},
$$

$s^{*}$ определяется формулой (2.2).

ДокАЗАТЕльСтво. Пусть $u, v$ - слабое решение задачи (4.1). Для любой финитной по переменной $r$ функции $\varphi \in W_{1}^{2}(K) \cap W_{2}^{1}(K),\left.\varphi\right|_{\partial K}=0$, из определения слабого решения с помошью интегрирования по частям получим

$$
\begin{aligned}
& \int_{K} v^{q} \varphi d x \leqslant-\int_{K} u \Delta \varphi d x \\
& \int_{K} u^{p} \varphi d x \leqslant-\int_{K} v \Delta \varphi d x .
\end{aligned}
$$

Для оценки интегралов справа применим неравенство Гёльдера (как в предельном случае $q=q^{*} \S 2$ ):

$$
\begin{aligned}
\int_{K} v^{q} \varphi d x & \leqslant-\int_{K} u \Delta \varphi d x=-\int_{\operatorname{supp}|\Delta \varphi|} u \varphi^{1 / p} \frac{\Delta \varphi}{\varphi^{1 / p}} d x \\
& \leqslant\left(\int_{\operatorname{supp}|\Delta \varphi|} u^{p} \varphi d x\right)^{1 / p}\left(\int_{\operatorname{supp}|\Delta \varphi|} \frac{|\Delta \varphi|^{p^{\prime}}}{\varphi^{p^{\prime}-1}} d x\right)^{1 / p^{\prime}} \\
& =\left(\int_{\operatorname{supp}|\Delta \varphi|} u^{p} \varphi d x\right)^{1 / p} J_{p^{\prime}}^{1 / p^{\prime}}, \\
\int_{K} u^{p} \varphi d x & \leqslant-\int_{K} v \Delta \varphi d x=-\int_{\operatorname{supp}|\Delta \varphi|} v \varphi^{1 / q} \frac{\Delta \varphi}{\varphi^{1 / q}} d x \\
& \leqslant\left(\int_{\operatorname{supp}|\Delta \varphi|} v^{q} \varphi d x\right)^{1 / q}\left(\int_{\operatorname{supp}|\Delta \varphi|} \frac{|\Delta \varphi|^{q^{\prime}}}{\varphi^{q^{\prime}-1}} d x\right)^{1 / q^{\prime}} \\
& =\left(\int_{\operatorname{supp}|\Delta \varphi|} v^{q} \varphi d x\right)^{1 / q} J_{q^{\prime}}^{1 / q^{\prime}}
\end{aligned}
$$

где

$$
J_{p^{\prime}}=\int_{\operatorname{supp}|\Delta \varphi|} \frac{|\Delta \varphi|^{p^{\prime}}}{\varphi^{p^{\prime}-1}} d x, \quad J_{q^{\prime}}=\int_{\operatorname{supp}|\Delta \varphi|} \frac{|\Delta \varphi|^{q^{\prime}}}{\varphi^{q^{\prime}-1}} d x .
$$

Подставим второе неравенство из (4.2) в правую часть первого, что даст

$$
\begin{aligned}
\int_{K} v^{q} \varphi d x & \leqslant\left\{\left(\int_{\operatorname{supp}|\Delta \varphi|} v^{q} \varphi d x\right)^{1 / q} J_{q^{\prime}}^{1 / q^{\prime}}\right\}^{1 / p} J_{p^{\prime}}^{1 / p^{\prime}} \\
& =\left(\int_{\operatorname{supp}|\Delta \varphi|} v^{q} \varphi d x\right)^{1 / q p} J_{q^{\prime}}^{1 / q^{\prime} p} J_{p^{\prime}}^{1 / p^{\prime}}
\end{aligned}
$$


Далее используем неравенство Юнга:

$$
\int_{K} v^{q} \varphi d x \leqslant \varepsilon \int_{\operatorname{supp}|\Delta \varphi|} v^{q} \varphi d x+\frac{1}{\varepsilon^{1 /(p q-1)}}\left(J_{q^{\prime}}^{1 / q^{\prime} p} J_{p^{\prime}}^{1 / p^{\prime}}\right)^{p q /(p q-1)},
$$

откуда получаем

$$
\int_{K} v^{q} \varphi d x \leqslant c_{0}\left(J_{q^{\prime}}^{1 / q^{\prime}} J_{p^{\prime}}^{p-1}\right)^{q /(p q-1)} .
$$

Аналогично подставляем первое неравенство из (4.2) в правую часть второго:

$$
\begin{aligned}
\int_{K} u^{p} \varphi d x & \leqslant\left\{\left(\int_{\operatorname{supp}|\Delta \varphi|} u^{p} \varphi d x\right)^{1 / p} J_{p^{\prime}}^{1 / p^{\prime}}\right\}^{1 / q} J_{q^{\prime}}^{1 / q^{\prime}} \\
& =\left(\int_{\operatorname{supp}|\Delta \varphi|} u^{p} \varphi d x\right)^{1 / q p} J_{p^{\prime}}^{1 / p^{\prime} q} J_{q^{\prime}}^{1 / q^{\prime}}
\end{aligned}
$$

откуда по неравенству Юнга находим

$$
\int_{K} u^{p} \varphi d x \leqslant \varepsilon \int_{\operatorname{supp}|\Delta \varphi|} u^{p} \varphi d x+\frac{1}{\varepsilon^{1 /(p q-1)}}\left(J_{p^{\prime}}^{1 / p^{\prime} q} J_{q^{\prime}}^{1 / q^{\prime}}\right)^{p q /(p q-1)}
$$

так что

$$
\int_{K} u^{p} \varphi d x \leqslant c_{0}\left(J_{p^{\prime}}^{1 / p^{\prime}} J_{q^{\prime}}^{q-1}\right)^{p /(p q-1)} .
$$

При $\varphi=\varphi_{\rho}$, где $\varphi_{\rho}$ определяется по формуле $(2.6)$ с $s=s_{*}$, для $J_{q^{\prime}}$ и $J_{p^{\prime}}$ согласно лемме 2.3 имеем

$$
J_{p^{\prime}} \leqslant \frac{C}{\rho^{2 p^{\prime}-s_{*}-N}}=\frac{C}{\rho^{2 p^{\prime}-s^{*}-2}}, \quad J_{q^{\prime}} \leqslant \frac{C}{\rho^{2 q^{\prime}-s_{*}-N}}=\frac{C}{\rho^{2 q^{\prime}-s^{*}-2}} .
$$

Подставим эти оценки в полученные выше неравенства. Тогда

$$
\begin{aligned}
& \int_{K \cap\{0<r<\rho\}} v^{q} \varphi_{\infty} d x \leqslant \int_{K} v^{q} \varphi_{\rho} d x \leqslant \frac{c_{5}}{\rho^{k_{1} q /(p q-1)}}, \\
& \int_{K \cap\{0<r<\rho\}} u^{p} \varphi_{\infty} d x \leqslant \int_{K} u^{p} \varphi_{\rho} d x \leqslant \frac{c_{5}}{\rho^{k_{2} p /(p q-1)}},
\end{aligned}
$$

где

$k_{1}=\frac{2 q^{\prime}-s^{*}-2}{q^{\prime}}+\left(2 p^{\prime}-s^{*}-2\right)(p-1), \quad k_{2}=\frac{2 p^{\prime}-s^{*}-2}{p^{\prime}}+\left(2 q^{\prime}-s^{*}-2\right)(q-1)$, $c_{5}$ не зависит от $\rho$.

Соотношения (4.3)-(4.5) полностью аналогичны соответствуюшим оценкам для случая одного неравенства из $\S 2$.

Следовательно, из оценок (4.5) вытекает, что если $k_{1} \geqslant 0$, то $v(x) \equiv 0$, если же $k_{2} \geqslant 0$, то $u(x) \equiv 0$. Заметим, что если хотя бы одна из функций $u(x)$ или $v(x)$ тождественно равна нулю, то равна нулю и другая. Действительно, если $v(x) \equiv 0$, то второе неравенство в определении обобщенного решения примет вид

$$
0 \geqslant \int_{K} u^{p} \varphi d x
$$


откуда $u(x) \equiv 0$, и наоборот.

Таким образом, условие отсутствия неотрицательного нетривиального решения задачи (4.1) имеет вид $\max \left\{k_{1}, k_{2}\right\} \geqslant 0$. После упрощения приходим к условию из формулировки

$$
\max \left\{\gamma_{1}, \gamma_{2}\right\} \geqslant \frac{s^{*}}{2}
$$

Теорема доказана.

Сравним теорему 4.1 и теорему 2.1 . Пусть в задаче (4.1) $q=p$. Тогда

$$
\gamma_{1}=\gamma_{2}=\frac{q+1}{q^{2}-1}=\frac{1}{q-1}
$$

и условие отсутствия нетривиальных решений теоремы 4.1 принимает вид

$$
\frac{1}{q-1} \geqslant \frac{s^{*}}{2}
$$

т.е. $q \leqslant 1+\frac{2}{s^{*}}$, что совпадает с аналогичным условием теоремы 2.1 .

Точность результата теоремы 4.1 покажем на примере функций

$$
u(r, \omega)=\frac{\varepsilon_{1}}{\left(1+r^{2}\right)^{\gamma_{1}}} \Phi(\omega), \quad v(r, \omega)=\frac{\varepsilon_{2}}{\left(1+r^{2}\right)^{\gamma_{2}}} \Phi(\omega),
$$

где $\gamma_{1}$ и $\gamma_{2}$ определены в формулировке теоремы $4.1, \varepsilon_{1}(q, p), \varepsilon_{2}(q, p)>0$ малы.

По аналогии с леммой 2.1 имеем

$$
\begin{aligned}
-\Delta u & =\frac{\varepsilon_{1} \Phi(\omega)}{\left(1+r^{2}\right)^{\gamma_{1}+1}}\left\{2 \gamma_{1} N-4 \gamma_{1}\left(\gamma_{1}+1\right) \frac{r^{2}}{1+r^{2}}+\lambda_{\omega} \frac{1+r^{2}}{r^{2}}\right\} \\
& \geqslant \frac{\varepsilon_{1} \Phi(\omega)}{\left(1+r^{2}\right)^{\gamma_{1}+1}} \delta_{1}(q, p), \\
-\Delta v & =\frac{\varepsilon_{2} \Phi(\omega)}{\left(1+r^{2}\right)^{\gamma_{2}+1}}\left\{2 \gamma_{2} N-4 \gamma_{2}\left(\gamma_{2}+1\right) \frac{r^{2}}{1+r^{2}}+\lambda_{\omega} \frac{1+r^{2}}{r^{2}}\right\} \\
& \geqslant \frac{\varepsilon_{2} \Phi(\omega)}{\left(1+r^{2}\right)^{\gamma_{2}+1}} \delta_{2}(q, p),
\end{aligned}
$$

где $\delta_{1}(q, p)>0, \delta_{2}(q, p)>0$, если $\gamma_{1}<s^{*} / 2$ и $\gamma_{2}<s^{*} / 2$, поскольку в этом случае выражения в фигурных скобках равномерно по $r>0$ положительны.

Далее, поскольку $\gamma_{1}+1=\gamma_{2} q$ и $\gamma_{2}+1=\gamma_{1} p$, а также учитьвая неравенства $\Phi(\omega) \geqslant \Phi^{q}(\omega)$ и $\Phi(\omega) \geqslant \Phi^{p}(\omega)$, получаем

$$
\begin{aligned}
& -\Delta u \geqslant \frac{\varepsilon_{1}}{\left(1+r^{2}\right)^{\gamma_{2} q}} \Phi^{q}(\omega) \delta_{1}(q, p)=v^{q}, \\
& -\Delta v \geqslant \frac{\varepsilon_{2}}{\left(1+r^{2}\right)^{\gamma_{1} p}} \Phi^{p}(\omega) \delta_{2}(q, p)=u^{p},
\end{aligned}
$$

если $\varepsilon_{1}=\delta_{1}^{1 /(q-1)}(q, p), \varepsilon_{2}=\delta_{2}^{1 /(p-1)}(q, p)$.

Таким образом, пара заданных так функций $u(x)$ и $v(x)$ является решением задачи (4.1), если $\gamma_{1}<s^{*} / 2$ и $\gamma_{2}<s^{*} / 2$, т.е.

$$
\max \left\{\gamma_{1}, \gamma_{2}\right\}<\frac{s^{*}}{2} .
$$

В заключение автор выражает благодарность С. И. Похожаеву за постановку задачи и полезное обсуждение результатов, а также В.В. Курте за постоянное внимание к работе. 


\section{Список литературы}

1. Kuzin I., Pohozaev S. Entire solutions of semilinear elliptic equations. Basel: Birkhäuser, 1997.

2. Митидиери Э., Похожаев С.И. Отсутствие глобальных положительных решений квазилинейных эллиптических неравенств // Докл. РАН. 1998. Т. 359. № 4. С. 456-460.

3. Митидиери Э., Похожаев С. И. Отсутствие положительных решений для систем квазилинейных эллиптических уравнений и неравенств в $\mathbb{R}^{N} / /$ Докл. РАН. 1999. Т. 366. № 1. C. $13-17$.

4. Митидиери Э., Похожаев С. И. Отсутствие положительных решений квазилинейных эллиптических задач в $\mathbb{R}^{N} / /$ Тр. МИАН. 1999. Т. 227. С. $192-222$.

5. Курта В.В. К вопросу об отсутствии целых положительных решений у полулинейных эллиптических уравнений // УМН. 1995. Т. 50. №4(304). С. 127.

6. Курта В. В. Некоторые вопросы качественной теории нелинейных дифференциальных уравнений второго порядка. Дис. ... доктора физ.-мат. наук. М.: МИАН, 1994.

7. Gidas B., Spruck J. Global and local behaviour of positive solutions of nonlinear elliptic equations // Comm. Pure Appl. Math. 1981. V. 34. P. 525-598.

8. Berestycki H., Capuzzo Dolcetta J., Nirenberg L. Superlinear indefinite elliptic problems and nonlinear Liouville theorems // Topological Methods in Nonlinear Analysis. 1995. V. 4. P. 59-78.

9. Bandle C., Essen $M$. On positive solutions of Emden equations in cone-like domains // Arch. Rational Mech. Anal. 1990. V. 112. № 4. P. 319-338.

10. Egnell H. Positive solutions of semilinear equations in cones // Trans. Amer. Math. Soc. 1992. V. 330. № 1. P. 191-201.

11. Нгуен Мань Хунг. Об отсутствии положительных решений нелинейных эллиптических уравнений второго порядка в конических областях // Дифференц. уравн. 1998. Т. 34. № 4. C. 533-539.

12. Bandle C. Positive solutions of Emden equations in a cone-like domains // Progress in Nonlinear Differential Equations and their applications. 1992. V. 7. P. 71-75.

13. Кондратьев B. А., Эйдельман C. Д. Положительные решения квазилинейных эллиптических уравнений второго порядка // Докл. РАН. 1994. Т. 334. № 4. С. 427-428.

14. Нгуен Мин Чи. Положительные решения уравнения Эмдена-Фоулера в конусе // Дифференц. уравн. 1994. Т. 30. № 4. С. 659-664.

15. Гольдштейн B. М., Решетняк Ю.Г. Введение в теорию функций с обобщенными производными и квазиконформные отображения. М.: Наука, 1983.

16. Мазья В. Г. Пространства С. Л. Соболева. Л.: Изд-во ЛГУ, 1985.

17. Миклюков B. М. Емкостные методы в задачах нелинейного анализа. Дис. ... доктора физ.-мат. наук. Тюмень: Тюменск. ГУ, 1980.

18. Похожаев С.И. Существенно нелинейные емкости, индуцированные парой дифференциальных операторов // Докл. РАН. 1997. Т. 357. № 5. С. 592-594.

Поступило в редакцию 18.V.1999 